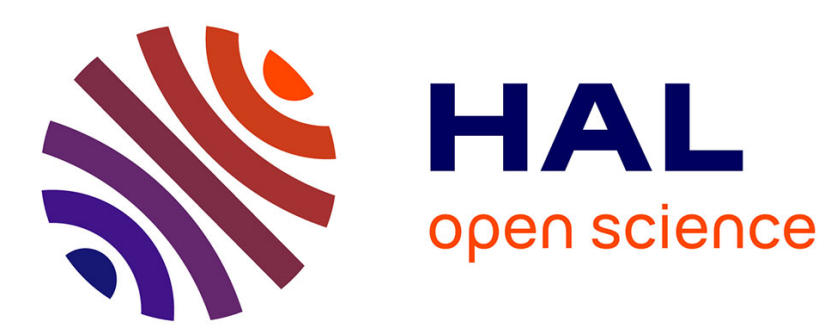

\title{
Spectres Raman du 2e ordre dans les cristaux mixtes Ga1- xAlxSb
}

F. Charfi, M. Zouaghi, A. Joullié, M. Balkanski, Ch. Hirlimann

\section{To cite this version:}

F. Charfi, M. Zouaghi, A. Joullié, M. Balkanski, Ch. Hirlimann. Spectres Raman du 2e ordre dans les cristaux mixtes Ga1- xAlxSb. Journal de Physique, 1980, 41 (1), pp.83-85. 10.1051/jphys:0198000410108300 . jpa-00209219

\section{HAL Id: jpa-00209219 https://hal.science/jpa-00209219}

Submitted on 1 Jan 1980

HAL is a multi-disciplinary open access archive for the deposit and dissemination of scientific research documents, whether they are published or not. The documents may come from teaching and research institutions in France or abroad, or from public or private research centers.
L'archive ouverte pluridisciplinaire HAL, est destinée au dépôt et à la diffusion de documents scientifiques de niveau recherche, publiés ou non, émanant des établissements d'enseignement et de recherche français ou étrangers, des laboratoires publics ou privés. 


\title{
Spectres Raman du $2^{\mathrm{e}}$ ordre dans les cristaux mixtes $\mathbf{G a}_{1-x} \mathbf{A l} \mathbf{l}_{x} \mathbf{S b}$
}

\author{
F. Charfi, M. Zouaghi, A. Joullie $\left({ }^{*}\right)$ M. Balkanski $\left({ }^{(*)}\right)$ et Ch. Hirlimann $\left({ }^{(*)}\right.$ \\ Laboratoire de Spectroscopie Moléculaire \\ Faculté des Sciences, Campus Universitaire Belvédère, Tunis (Tunisie)
}

(Reçu le 7 mai 1979, révisé le 10 août, accepté le 17 septembre 1979)

\begin{abstract}
Résumé. - Les spectres de diffusion Raman du deuxième ordre du cristal mixte $\mathrm{Ga}_{1-x} \mathrm{Al}_{\boldsymbol{x}} \mathrm{Sb}$ ont été mesurés pour $0<x<1$. L'évolution des combinaisons de phonons avec la concentration a permis de mettre en évidence un comportement à un mode pour les phonons acoustiques et un comportement à deux modes pour les phonons optiques. Les spectres font apparaître des combinaisons de phonons optiques du type GaSb $+\mathrm{AlSb}$.
\end{abstract}

\begin{abstract}
The second order Raman spectra of the mixed crystal $\mathrm{Ga}_{1-x} \mathrm{Al}_{x} \mathrm{Sb}$ have been measured for $0<x<1$. The combinations of phonons as a fonction of concentration have been investigated. A one-mode behaviour for the acoustical phonons and a two-mode behaviour for the optical phonons are found. In addition, combinations of optical GaSb + AlSb like modes have been emphasized.
\end{abstract}

1. Introduction. - Les propriétés vibratoires des cristaux mixtes ont suscité un grand intérêt sur le plan expérimental et théorique ces dix dernières années. Les mesures par spectroscopie Raman et par réflectivité dans l'infrarouge ont permis d'étudier les modes de vibration de centre de zone d'un grand nombre de cristaux mixtes. Plusieurs modèles ont été proposés pour interpréter le comportement à un mode ou à deux modes des alliages : modèles « R.E.I. " (randomelement isodeplacement) [1]; modèle d'agrégats [2]; modèle de la pseudo-cellule [3] ; modèles basés sur la technique des fonctions de Green [4]; modèle "C.P.A. " (coherent potentiel approximation) [5].

L'étude des alliages $\mathrm{Ga}_{1-x} \mathrm{Al}_{x} \mathrm{Sb}$ par réflectivité dans l'infrarouge [6, 7] et par spectroscopie Raman [8] a mis en évidence le comportement à deux modes des phonons de petit vecteur d'onde. Nous présentons les spectres Raman du deuxième ordre des alliages $\mathrm{Ga}_{1-x} \mathrm{Al}_{x} \mathrm{Sb}$ et nous nous proposons d'interpréter leur évolution avec la concentration $x$.

2. Résultats expérimentaux. - L'échantillon de $\mathrm{GaSb}$ pur est un monocristal obtenu par la méthode de Czochralsky. Tous les autres échantillons ont été élaborés par la méthode de Bridgman et sont polycristallins.

$\left(^{*}\right)$ Centre d'Etude d'Electronique des Solides, Université des Sciences et Techniques du Languedoc, Montpellier (France).

(**) Laboratoire de Physique des Solides, Université P.-etM.-Curie, 4, place Jussieu, 75005 Paris (France).
Les spectres Raman ont été obtenus à la température ambiante avec une longueur d'onde excitatrice égale à $514,5 \mathrm{~nm}$.

Le spectre Raman de GaSb pur est représenté sur la figure $1 a$. Les bandes situées de part et d'autre des pics $\mathrm{LO}(\Gamma)$ et $\mathrm{TO}(\Gamma)$ sont dues à une diffusion Raman du deuxième ordre. La comparaison du spectre de diffusion du deuxième ordre et la courbe de densité d'états du GaSb [9] permet d'attribuer la bande de basse fréquence à des combinaisons de phonons acoustiques et celle située à haute fréquence à des combinaisons de phonons optiqués [10].

Le spectre Raman du composé pur AlSb est représenté sur la figure $1 b$. Etant donné que les mesures de diffusion inélastique des neutrons n'ont pas été faites sur le composé AlSb, nous disposons de peu d'information sur les phonons de bord de zone [1]. L'attribution des pics observés dans le spectre Raman du deuxième ordre de AlSb ne peut être qu'approximative.

La figure 2 représente les spectres Raman du deuxième ordre situés à basse fréquence de $\mathrm{GaSb}$, $\mathrm{AlSb}$ et quelques alliages $\mathrm{Ga}_{1-x} \mathrm{Al}_{x} \mathrm{Sb}$. $\mathrm{La}$ bande de basse fréquence évolue d'une façon continue jusqu'à une concentration $x$ de l'ordre de 0,14 . Quand la concentration $x$ augmente $(x>0,2)$, la bande se présente différemment et les mesures des spectres Raman en fonction de la température ont montré qu'elle ne peut pas être attribuée à un processus de diffusion du deuxième ordre [12].

Les spectres Raman du deuxième ordre situés à haute fréquence, obtenus pour différentes valeurs de 


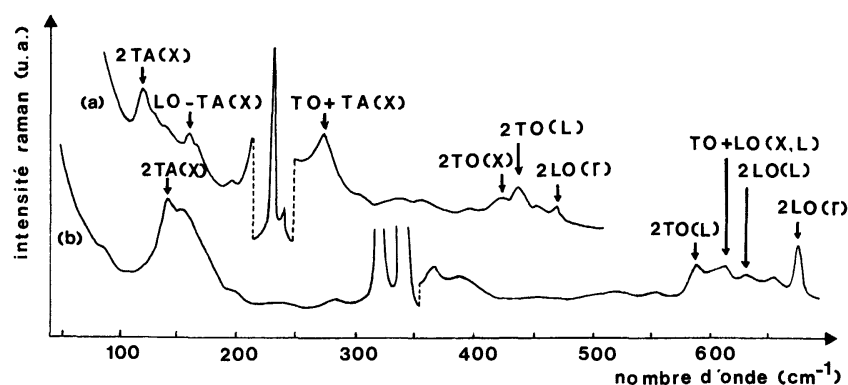

Fig. 1. - Spectre Raman du $1^{\mathrm{er}}$ ordre et du deuxième ordre de GaSb pur (a) et de AlSb pur (b).

[First- and second-order Raman spectra of pure GaSb (a) and pure AlSb $(b)$.]

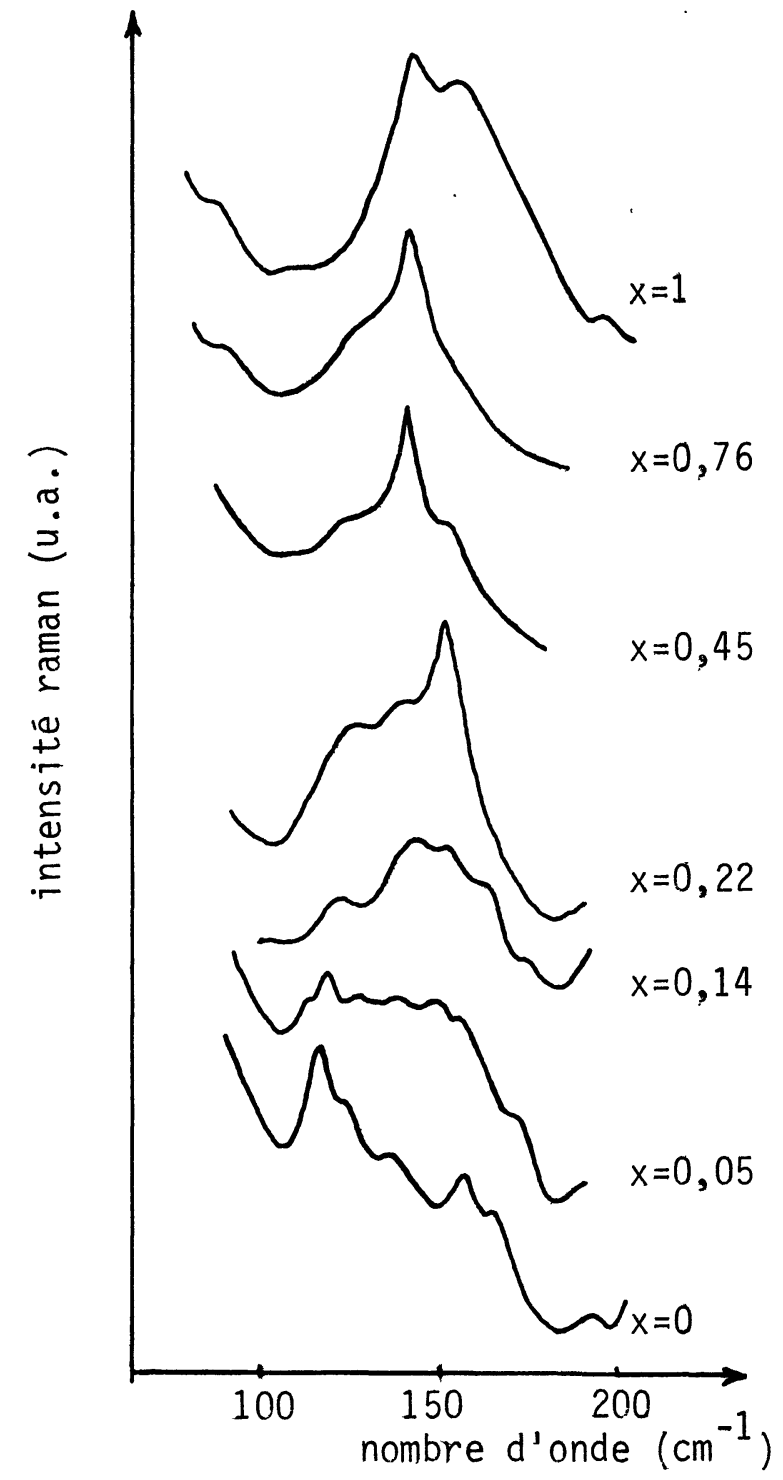

Fig. 2. - Evolution du spectre Raman du deuxième ordre à basse fréquence des alliages $\mathrm{Ga}_{1-x} \mathrm{Al}_{x} \mathrm{Sb}$ avec la concentration.

[The effect of the introduction of aluminium on the low frequency second-order Raman spectra of $\mathrm{Ga}_{1-x} \mathrm{Al}_{x} \mathrm{Sb}$.]

la concentration $x$ sont représentés sur la figure 3 . A partir des concentrations limites $(x=0, x=1)$, nous pouvons suivre par continuité les bandes attri- buées à des combinaisons de phonons optiques de centre de zone et de bord de zone, relatives à chacun des constituants purs. Il faut noter que les bandes ont des structures moins prononcées dans l'alliage que dans le constituant pur. D'autre part le domaine de fréquence de la bande relative au mode AlSb $\left(580 \mathrm{~cm}^{-1}-680 \mathrm{~cm}^{-1}\right)$ se réduit lorsque la concentration en aluminium diminue; la même variation est observée pour la bande relative au mode $\mathrm{GaSb}$ $\left(410 \mathrm{~cm}^{-1}-470 \mathrm{~cm}^{-1}\right)$.

Une troisième bande apparaît dans un domaine de fréquences compris entre $510 \mathrm{~cm}^{-1}$ et $560 \mathrm{~cm}^{-1}$; le maximum d'intensité de cette bande est observé pour $x$ de l'ordre de 0,5 .

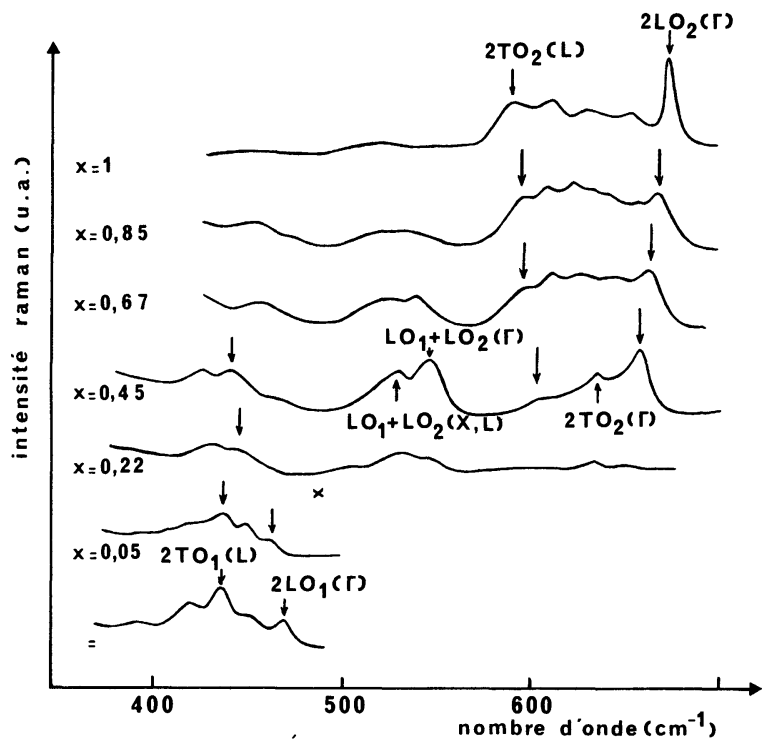

Fig. 3. - Spectres Raman du deuxième ordre à haute fréquence des alliages $\mathrm{Ga}_{1-x} \mathrm{Al}_{x} \mathrm{Sb}$; les indices 1 et 2 sont relatifs respectivement aux modes du type GaSb et AlSb.

[High frequency second-order Raman spectra in the alloys $\mathrm{Ga}_{1-x} \mathrm{Al}_{x} \mathrm{Sb}$ : the index 1 and 2 correspond respectively to the $\mathrm{GaSb}$ like mode and AlSb like mode.]

3. Discussion. - L'étude des alliages $\mathrm{Ga}_{1-{ }_{x}} \mathrm{Al}_{x} \mathrm{Sb}$ par spectroscopie Raman [8] et par réflectivité dans l'infrarouge $[6,7]$ met en évidence deux groupes de fréquences caractéristiques de chacun des composés purs $\mathrm{GaSb}$ et $\mathrm{AlSb}$. Le comportement à deux modes des phonons optiques de petit vecteur d'onde est déduit de façon satisfaisante, à partir de modèles se basant essentiellement sur les variations de masse. En effet, le paramètre cristallin ne varie que de $0,3 \%$ entre les concentrations extrêmes $x=0$ et $x=1$ et les constantes de force sont peu modifiées lorsque $x$ varie.

Par conséquent, l'évolution de la densité d'états à un phonon dans les alliages $\mathrm{Ga}_{1-x} \mathrm{Al}_{x} \mathrm{Sb}$ est déterminée principalement par la différence de masse des atomes se substituant $\mathrm{Ga}$ et $\mathrm{Al}$. La courbe de densité d'états contient une bande à basse fréquence dominée par les vibrations de l'atome le plus lourd Sb, donc peu 
sensible aux variations de la concentration $x$, et un spectre à haute fréquence relatif aux vibrations de $\mathrm{Ga}$ et Al. Etant donné la différence de masse entre ces deux atomes, le spectre de haute fréquence comprend deux bandes pour $x \neq 0$ et $x \neq 1$ d'intensité relative approximativement proportionnelle à $\frac{x}{1-x}$.

Etant donné que le spectre Raman du deuxième ordre reflète la densité d'états à deux phonons, le modèle précédent permet d'interpréter son évolution avec la concentration $x$. La bande de basse fréquence qui apparaît pour $x<0,2$ (Fig. 2) peut être attribuée à des combinaisons de phonons acoustiques en supposant que les combinaisons de type acoustique-optique sont peu actives; la faible variation de fréquence observée correspond au comportement prévu pour les modes acoustiques.

Les spectres Raman du deuxième ordre situés à haute fréquence (Fig. 3) comportent pour $x \neq 0$ et $x \neq 1$, trois bandes :

1) La bande située entre $410 \mathrm{~cm}^{-1}$ et $470 \mathrm{~cm}^{-1}$ est attribuée à des combinaisons de phonons optiques de centre de zone et de bord de zone du type GaSb. Quand la concentration en gallium diminue, la largeur de la bande se réduit autour de la fréquence $420 \mathrm{~cm}^{-1}$ : cette valeur correspond en effet au double de la fréquence du mode d'impureté $\mathrm{Ga}$ dans $\mathrm{AlSb}$ vers lequel tendent tous les modes optiques du type $\mathrm{GaSb}$.
2) La bande située entre $580 \mathrm{~cm}^{-1}$ et $680 \mathrm{~cm}^{-1}$ est attribuée à des combinaisons de phonons optiques de centre de zone et de bord de zone du type AlSb. Lorsque la concentration $x$ en aluminium diminue, le domaine de fréquence de la bande se réduit du fait de la dégénérescence de tous les modes optiques en un mode d'impureté à la limite $x=0$.

3) La bande intermédiaire $\left(510 \mathrm{~cm}^{-1}-560 \mathrm{~cm}^{-1}\right)$ pourrait correspondre à des combinaisons de phonons optiques du type GaSb et AlSb.

4. Conclusion. - L'étude des spectres Raman du deuxième ordre du cristal mixte $\mathrm{Ga}_{1-x} \mathrm{Al}_{x} \mathrm{Sb}$ a permis de mettre en évidence un comportement à un mode pour les phonons acoustiques et un comportement à deux modes pour les phonons optiques. Malgré le manque de données sur les modes de vibrations aux points de haute symétrie de la zone de Brillouin du composé pur AlSb et le caractère polycristallin des échantillons, certains pics du deuxième ordre ont pu être attribués. Des mesures de spectres Raman en polarisation sur des échantillons monocristallins de AlSb et sur des alliages épitaxiés nous permettront de confirmer les résultats obtenus et de compléter l'attribution des pics du deuxième ordre.

Remerciements. - Nous remercions Monsieur C. Llinares pour sa collaboration fructueuse.

\section{Bibliographie}

[1] Chen, Y. S., Shokley, W. et Pearson, G. L., Phys. Rev. 151 (1966) 648

[2] Verleur, H. W. et Barker, Jr. A. S., Phys. Rev. 149 (1966) 715.

[3] Varshney, S. C., Vetelino, J. F., Mitra, S. S. et Chang, I. F., Phys. Rev. B 12 (1975) 5912.

[4] Beserman, R. et Balkanski, M., Phys. Rev. B 1 (1970) 608.

[5] TAYloR, D. W., Phys. Rev. 156 (1967) 809.

[6] Lucovsky, G., Cheng, K. Y., Pearson, G. L., Phys. Rev. B 12 (1975) 4135

[7] Ipatova, I. P., IChKitiDZE, R. R., Sochil INA, I. N., UkHANOv,
Yu. I. et Shmartsev Yu. V., Sov. Phys. Solid State 18 (1976) 306.

[8] Charfi, F., Zouaghi, M., Llinares, C., Balkanski, M., Hirlimann, Ch. et Joullie, A., Proc. Int. Conf. Lattice Dynamics Balkanski (Paris 1977) 438.

[9] Farr, M. K., Traylor, J. G. et Sinha, S. K., Phys. Rev. B 11 (1975) 1587.

[10] Klein, P. B. et Chang, R. K., Phys. Rev. B 14 (1976) 2498.

[11] Turner, W. J. et Reese, W. E., Phys. Rev. 127 (1962) 126.

[12] Charfi, F., Thèse $3^{e}$ Cycle, Tunis (1978). 